NIST Technical Note 2146

\title{
Recalibration Intervals for Radiation Detection Instruments
}

L. Pibida

B. Estes

M. Mejias

G. Klemic

This publication is available free of charge from:

https://doi.org/10.6028/NIST.TN.2146

National Institute of Standards and Technology U.S. Department of Commerce 


\title{
Recalibration Intervals for Radiation Detection Instruments
}

\author{
L. Pibida \\ Radiation Physics Division \\ Physical Measurement Laboratory \\ B. Estes \\ M. Mejias \\ Gaithersburg Radiation Safety Division \\ Office of Safety, Health, and Environment
}

\author{
G. Klemic \\ National Urban Security Technology Laboratory \\ Department of Homeland Security
}

This publication is available free of charge from:

https://doi.org/10.6028/NIST.TN.2146

April 2021

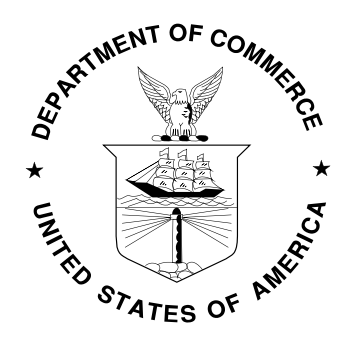

U.S. Department of Commerce

Gina M. Raimondo, Secretary

National Institute of Standards and Technology James K. Olthoff, Performing the Non-Exclusive Functions and Duties of the Under Secretary of Commerce for Standards and Technology \& Director, National Institute of Standards and Technology 
Certain commercial entities, equipment, or materials may be identified in this document in order to describe an experimental procedure or concept adequately. Such identification is not intended to imply recommendation or endorsement by the National Institute of Standards and Technology, nor is it intended to imply that the entities, materials, or equipment are necessarily the best available for the purpose.

National Institute of Standards and Technology Technical Note 2146 Natl. Inst. Stand. Technol. Tech. Note 2146, 25 pages (April 2021) CODEN: NTNOEF

This publication is available free of charge from: https://doi.org/10.6028/NIST.TN.2146 


\begin{abstract}
Many state and local emergency management and public health agencies have radiation detection equipment for homeland security preparedness and response efforts. Such equipment is not used for routine health physics or regulatory compliance measurements, and while some instrument manufacturers provide recommendations for periodic calibrations, general guidance on appropriate performance maintenance is lacking. In contrast, institutional health physics programs for facilities licensed by the Nuclear Regulatory Commission (NRC) may have calibration intervals specified in their license, for example with a requirement for $20 \%$ accuracy and annual laboratory calibrations [1]. Annual laboratory calibration intervals may not be practical or appropriate for homeland security emergency applications that are not covered by NRC regulations. In this work we provide a summary of the NCSL RP-1 calibration interval analysis method, and examples of the method applied to three sets of available data (two models of radiation survey meters and an electronic dosimeter). Information is also provided on how to develop control charts to monitor individual instruments' performance.
\end{abstract}

\title{
Key words
}

Radiation detector calibration, calibration intervals, control charts, application of NCSL RP-1 method. 


\section{Table of Contents}

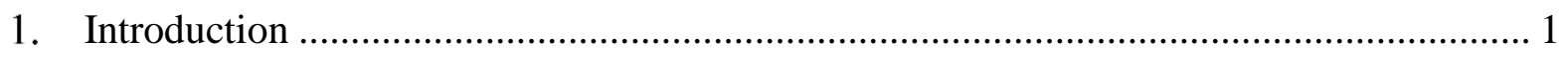

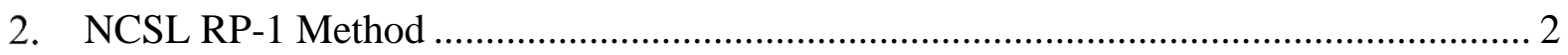

2.1. Sensitivity Analysis of NCLS RP-1 Input........................................................... 4

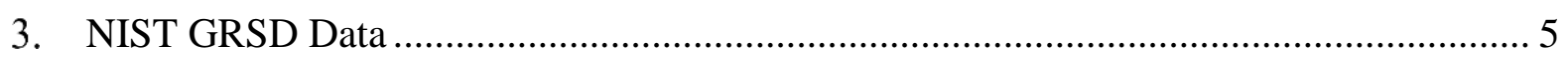

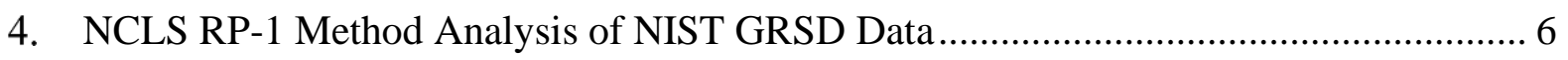

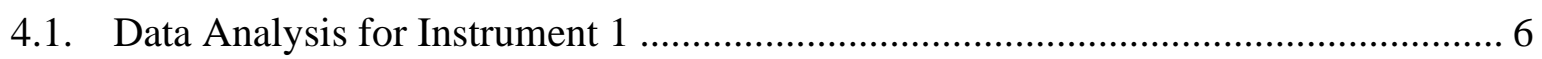

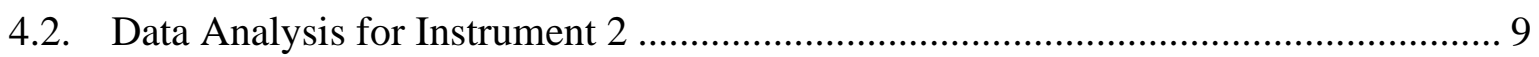

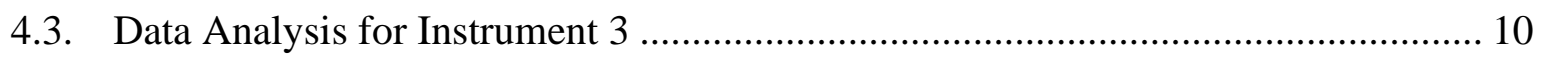

4.4. Exposure Rate Readings for Instrument 2 ……………...................................... 11

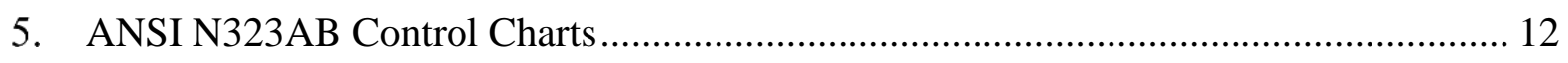

5.1. Examples of control charts for instrument providing exposure or dose rate or count

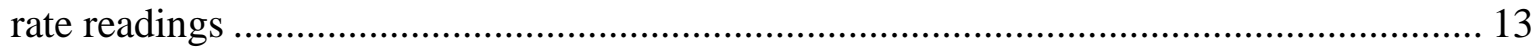

5.2. Examples of control charts and plots for instrument with radionuclide identification

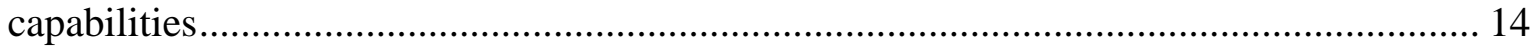

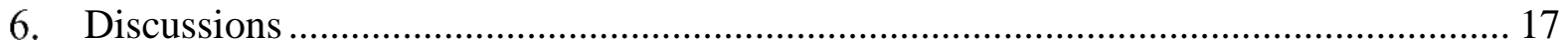

6.1. For instruments providing dose, exposure or count rate readings............................ 17

6.2. For instrument with radionuclide identification capabilities...................................... 17

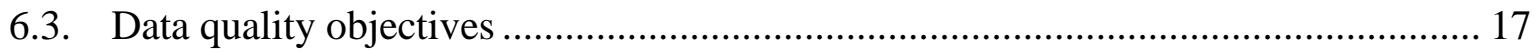

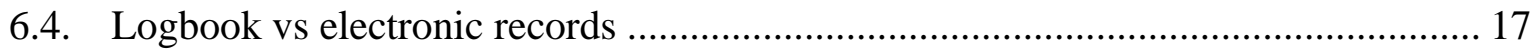

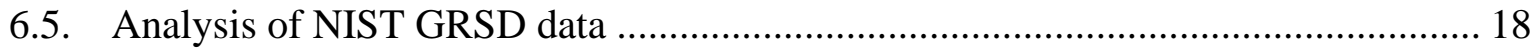

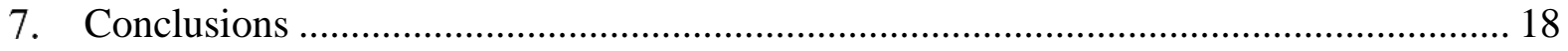

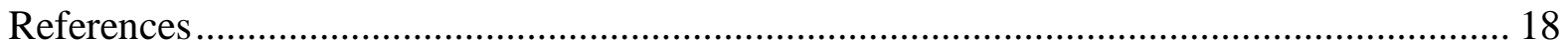

\section{List of Tables}

Table 1: Example cases showing the behavior of the NCSL RP-1 tool .................................... 4

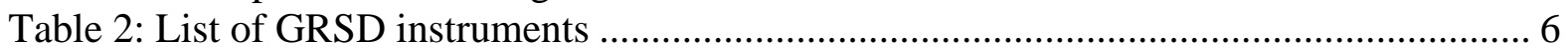

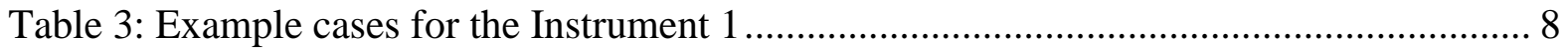

\section{List of Figures}

Fig. 1: Instrument 1 in-tolerance readings as a function of time for 3.71 years for all units. The different color symbols represent the different units. There are 10 dose rate values per calibration for each unit. The gray triangle at zero values, is one exposure rate value out-oftolerance while the other 9 values are in tolerance. 
Fig. 2: Instrument 2 in tolerance readings as a function of time for 3.96 years for all units. The different color symbols represent the different units. There are 11 dose rate values per calibration for each unit. 10

Fig. 3: Instrument 3 in tolerance readings as a function of time for 3.14 years for all units. The different color symbols represent the different units. There are 8 dose rate values per calibration for each unit. 11

Fig. 4: Exposure rate difference between the instrument measured value and the radiation field used for the measurements expressed in percent. The lines represent the $0 \%$, the $\pm 20 \%$ and the $\pm 30 \%$ differences. The points outside the $\pm 20 \%$ lines and at $-90 \%$ represent some of the out-of-tolerance values. 12 Fig. 5: Full energy peak efficiency for the $122 \mathrm{keV}$ gamma-ray from the ${ }^{57} \mathrm{Co}$ emission. The triangles represent the measured values over time for the year 2019. The time zero and the solid line represent the initial measurement performed in 2009. The dotted lines represent the $\pm 1.5 \%$ values of the initial value.

Fig. 6: Full energy peak efficiency for the $1332 \mathrm{keV}$ gamma-ray from the ${ }^{60} \mathrm{Co}$ emission. The triangles represent the measured values over time for the year 2019. The time zero and the solid line represent the initial measurement performed in 2009. The dotted lines represent the $\pm 1.5 \%$ values of the initial value. 15

Fig. 7: Energy difference between the measured and evaluated values for the main gamma-ray lines for ${ }^{57} \mathrm{Co}$ and ${ }^{60} \mathrm{Co}$. The solid line represents zero difference. 16 Fig. 8: Full width half maximum (FWHM) for the main gamma-ray lines for ${ }^{57} \mathrm{Co}$ and ${ }^{60} \mathrm{Co}$. 16 


\section{Introduction}

Many state and local emergency management and public health agencies have radiation detection equipment for homeland security preparedness and response efforts. Such equipment is not used for routine health physics or regulatory compliance measurements, and while some instrument manufacturers provide recommendations for periodic calibrations, general guidance on appropriate performance maintenance is lacking. In contrast, institutional health physics programs for facilities licensed by the Nuclear Regulatory Commission (NRC) may have calibration intervals specified in their license, for example with a requirement for $20 \%$ accuracy and annual laboratory calibrations [1]. Annual laboratory calibration intervals may not be practical or appropriate for homeland security emergency applications that are not covered by NRC regulations. The National Council on Radiation Protection and Measurements (NCRP) Scientific Committee 3-2 is currently developing an NCRP statement to address the calibration of such emergency radiation detection equipment.

Calibration refers to the process of comparing an instrument indication with the reference value of a known radiation field: if the reading is found to be outside of an acceptable range, adjustments are made to the device; if within the acceptance range, no adjustments are made. Methods for determining appropriate calibration intervals based on observed instrument performance have been published by the American National Standards Institute (ANSI) and the National Conference of Standards Laboratories (NCSL). Some of those methods are presented here, applied to historical data from a health physics calibration lab, as a test case to analyze and illustrate the methods and to inform the NCRP effort.

At the National Institute of Standards and Technology (NIST) Gaithersburg Radiation Safety Division (GRSD) many instruments are being used and calibrated to ensure safety of personnel and protection of the environment. Some of the instruments used at NIST are similar to those used by many organizations for detection and preparedness/response efforts. GRSD has electronic records of the instruments' calibrations dating from 2016 in addition to having many units of the same instrument model. Prior to this, all records were kept for decades in paper form (e.g. logbooks). Data from the electronic records were used to perform an analysis of the GRSD current calibration intervals by applying the method described in the National Conference of Standards Laboratories (NCSL) International (2010), Recommended Practice RP-1: Establishment and Adjustment of Calibration Intervals [2].

The American National Standard for Radiation Protection Instrumentation Test and Calibration, Portable Survey Instruments, ANSI N323AB [3], provides additional methods to determine the calibration intervals, including the use of control charts to track individual instrument performance over time using periodic source response checks. A subset of the GRSD data used in the NCSL RP-1 analysis is used to illustrate this ANSI N323AB method.

In this work we provide a summary of the NCSL RP-1 calibration interval analysis method, and examples of the method applied to three sets of available data (two models of radiation survey meters and an electronic dosimeter). Information is also provided on how to develop control charts to monitor individual instruments' performance. Over time a control chart can aid in determining when an instrument requires recalibration; an example is provided for a gamma-ray spectrometer. The results presented here do not cover consideration of the costs 
involved in the optimization assessment of the calibration interval analysis. The NCSL RP-1 document provides information about the relative cost of performing this type of analysis depending on the number of devices that require calibration.

\section{NCSL RP-1 Method}

The NCSL RP-1 report provides a variety of possible methods to determine the optimal instrument or device calibration interval based on the number of instruments that require calibration, the funding available to perform these calibrations and the regulatory requirements that may apply to these instruments based on their use. It is designed to be applied to a pooled data set of periodic measurement results from multiple units of the same model of instrument. This method is fully described in [2], a summary of how this method evaluates the calibration interval is as follows: the "current interval" is tested by comparing the "Observed Reliability" to the "Reliability Target" of the instrument to see if there is a significant difference between the two. The reliability refers to the probability that an instrument reading is in-tolerance. The Observed Reliability $R_{o b s}=$ Number in tolerance/number of tests. The magnitude of this difference and the "Test Confidence Level" determine whether or not the difference is significant. If so, the current interval is rejected, and an adjusted interval is recommended. The upper and lower confidence limits are calculated for the measured data using a binomial distribution function. The "Interval Rejection Confidence" is the confidence of rejecting the "Current Interval". If the "Reliability Target" lies above the upper or below the lower confidence limits the "Current Interval" is rejected and a new interval is calculated. The newly recommended interval may be shorter than (or stay the same as) the current interval if there is a large number of instrument readings that are out-of-tolerance, or a longer interval may be recommended if the instrument readings are in-tolerance for a longer period of time compared to the current calibration interval.

There is a tool available online that can be used to evaluate data using this method. This tool can be found at: http://www.isgmax.com/calint_freeware.htm [4]. The tool involves the following input and output parameters:

1) Input: User-defined Administrative Criteria (or constraints)

- Reliability Target $\left(R_{\text {targ }}\right)$ : probability that an instrument reading is in-tolerance (intolerance goal), measurement reliability consistent with mission/use requirements and logistics and economic constraints

- Out-of-Tolerance Rate Target: the percent of instruments that can be out-oftolerance and still use the instruments without recalibration, it is equal to $100-R_{\text {targ }}$ (calculated by the program)

- Display Precision: number of digits used to display the calculated recommended interval

- Minimum Allowed Interval: the minimum calibration interval ( 0 can be used as the minimum)

- Maximum Allowed Interval: a value larger than the time for which there is available data is used here for the method to evaluate a longer calibration interval 
- Interval Change Confidence: represents the confidence with which an interval is rejected as yielding an observed reliability or out-of-tolerance rate that is noncompliant with the reliability target.

2) Input: Observed Data (from calibration measurements)

- Current Interval: time between measurements of instrument response (if measurements were out of tolerance at times shorter than the current calibration interval) or time to measurement found to be first out of tolerance (if measurements were out of tolerance at times longer than the current calibration interval)

- Longest Interval: time to first out-of-tolerance reading or time range of available data if all are in-tolerance, i.e. the longest observed time until instrument adjustment (recalibration) was needed

- Number of Tests: number of available measurements, summed over all instruments of the same model

- Number In-Tolerance: number of in-tolerance measurements

- Number Out-of-Tolerance: calculated by the program from the 2 values above (number of tests - number in-tolerance)

- Observed Reliability $\left(R_{o b s}\right)$ : in-tolerance / number of tests calculated from the above input data

- Observed Out-of-Tolerance Rate: out-of-tolerance / number of tests calculated from the above input data

3) Output: Test Results (calculated by the method)

- Interval Rejection Confidence: this is the confidence for rejecting the Current Interval

- Upper Confidence Limit: this is the value of the upper confidence limit that corresponds to the entered Interval Change Confidence

- Lower Confidence Limit: this is the value of the lower confidence limit that corresponds to the entered Interval Change Confidence

- Recommended Interval: output value calculated by the program

The reliability target value selected will depend on the mission/user requirement and logistics and economic constraints, it is up to the instrument user to select this value. To illustrate how the method works, the NIST data were analyzed using a reliability target of $95 \%$ and an interval change confidence of $95 \%$.

When using this tool [4] if the Current Interval is rejected, a new recommended interval is computed using a simple algorithm. The process takes place in two stages. In the first stage, the interval is adjusted to a trial value. In the second stage, the trial value is refined to ensure its feasibility and compliance with the user-defined administrative criteria or constraints entered as part of the criteria (i.e., Minimum Allowed Interval, Maximum Allowed Interval, Interval Change Confidence). 


\subsection{Sensitivity Analysis of NCLS RP-1 Input}

Results vary depending on the number of measurements available and the user-defined parameters listed above, this is illustrated in Case studies 5 and 6 show that the user-settings for maximum and minimum allowed intervals also affect whether an interval is rejected or not. For example, using a maximum allowed interval of 10,000 days, and 50 in-tolerances observed out of 50 tests, the recommended interval will be 144 days. Given the same circumstances with a maximum allowed interval of 120 days (smaller than the longest interval), the recommended interval will be 120 days [4].

Table 1 using various case studies. In each case, the following input parameters were held constant, set as follows: Minimum allowed interval $=0$ days, Current interval $=90$ days, and Longest interval $=200$ days. In all cases, all of the tests were in-tolerance (Observed Reliability $=100 \%)$, but in some cases the number of tests was varied.

Varying the Interval Change Confidence can yield interesting results. Case studies 1 and 2 show that the number of tests required to have a recommendation to lengthen the interval changes from 36 test to 19 tests when the Interval Change Confidence is changed from $95 \%$ to $70 \%$. Recommendations to increase the interval occurs for a smaller number of tests for lower Interval Change Confidence values [4].

Case studies 3 and 4 show that for a higher reliability target, a greater number of tests are needed to lengthen an interval, even if all tests are in-tolerance. For example, using the same fixed parameters as discussed above (with an Interval Change Confidence of $95 \%$ ), and a reliability target of $R_{\text {targ }}=95 \%$, a recommendation for changing the current interval will occur for 72 tests (with 72 tests in-tolerance). If $R_{\text {targ }}=64 \%$, then a recommendation for changing the current interval will occur when 9 in-tolerances are observed out of 9 tests [4].

The opposite is true for shortening intervals. For example, using the same parameter as discussed above, suppose that $R_{\text {targ }}=95 \%$ and the Interval Change Confidence is $95 \%$. A recommendation for not changing the current interval will occur if 71 in-tolerances are observed out of 71 tests. If the Interval Change Confidence is $95 \%$, but $R_{\text {targ }}=64 \%$, then a recommendation for not changing the current interval will occur when 8 in-tolerances are observed out of 8 tests [4].

Case studies 5 and 6 show that the user-settings for maximum and minimum allowed intervals also affect whether an interval is rejected or not. For example, using a maximum allowed interval of 10,000 days, and 50 in-tolerances observed out of 50 tests, the recommended interval will be 144 days. Given the same circumstances with a maximum allowed interval of 120 days (smaller than the longest interval), the recommended interval will be 120 days [4].

Table 1: Example cases showing the behavior of the NCSL RP-1 tool

\begin{tabular}{|l|l|l|l|l|l|l|}
\hline & Case 1 & Case 2 & Case 3 & Case 4 & Case 5 & Case 6 \\
\hline $\boldsymbol{R}_{\text {targ }}$ & $90 \%$ & $90 \%$ & $95 \%$ & $64 \%$ & $90 \%$ & $90 \%$ \\
\hline Min allowed interval & 0 days & 0 days & 0 days & 0 days & 0 days & 0 days \\
\hline
\end{tabular}




\begin{tabular}{|l|l|l|l|l|l|l|}
\hline Max allowed interval & 10000 days & 10000 days & 10000 days & 10000 days & 10000 days & 120 days \\
\hline $\begin{array}{l}\text { Interval change } \\
\text { confidence }\end{array}$ & $95 \%$ & $70 \%$ & $95 \%$ & $95 \%$ & $95 \%$ & $95 \%$ \\
\hline Current interval & 90 days & 90 days & 90 days & 90 days & 90 days & 90 days \\
\hline Longest interval & 200 days & 200 days & 200 days & 200 days & 200 days & 200 days \\
\hline $\begin{array}{l}\text { Number of tests } \\
\text { Number in-tolerance }\end{array}$ & 36 & 19 & 72 & 9 & 50 & 50 \\
\hline $\begin{array}{l}\text { Observed reliability } \\
\text { confidence }\end{array}$ & $100 \%$ & $100 \%$ & $100 \%$ & $100 \%$ & $100 \%$ & $100 \%$ \\
\hline $\begin{array}{l}\text { Interval rejection } \\
\text { limit }\end{array}$ & $97.75 \%$ & $86.49 \%$ & $97.51 \%$ & $98.20 \%$ & $99.49 \%$ & $99.49 \%$ \\
\hline $\begin{array}{l}\text { Lower confidence } \\
\text { limit }\end{array}$ & $90.26 \%$ & $90.50 \%$ & $95.01 \%$ & $66.37 \%$ & $92.89 \%$ & $92.89 \%$ \\
\hline $\begin{array}{l}\text { Recommended } \\
\text { interval }\end{array}$ & 180 days & 150 days & 180 days & 180 days & 144 days & 120 days \\
\hline
\end{tabular}

\section{NIST GRSD Data}

GRSD historical data were available for 9 different instrument models, but only 3 had sufficiently large datasets of greater than 20 data points (combined number of instrument units and measurement points) suitable to apply the NCSL RP-1 methodology. For illustrating the use of the NCSL RP-1 method, the 3 instruments with the largest numbers of units were selected independent of their performance and type. The information about these instruments is listed in Table 2. The NIST data has the following characteristics:

- The data was recorded in an electronic database starting on March 2016. This date corresponds to time zero in the figures below.

- Current interval is 1 year [1]. Different intervals were used to determine the optimal calibration intervals depending on the available data (see data in Sec. 4 below).

- In some cases, additional measurements were performed within 1 year (smaller time intervals).

- For each yearly measurement, the instruments were exposed to several dose rate values for each displayed decade depending on the instrument measurement range (in some cases measurements were performed at the lower and upper end of each decade depending on the type of instrument).

- Instruments were recalibrated (adjusted) only if the readings were out of range. 
- The GRSD acceptance range was $\pm 20 \%$ (meaning that the instrument was considered out of range and adjusted if the instrument reading were outside this range for each reference calibration value).

- The measurements were performed using the calibrated NIST ${ }^{137} \mathrm{Cs}$ irradiators. The number of exposure or dose rate values used to perform the measurements depended on the measurement range of each instrument.

- The Instrument 2 instruments were adjusted in some cases when the readings were within the acceptance range, in these cases the measurements were recorded as out-oftolerance.

Table 2: List of GRSD instruments

\begin{tabular}{|l|c|c|l|l|}
\hline $\begin{array}{l}\text { Instrument } \\
\text { model }\end{array}$ & $\begin{array}{l}\text { \# of units } \\
\text { per model }\end{array}$ & $\begin{array}{l}\text { \# of years } \\
\text { of data }\end{array}$ & $\begin{array}{l}\text { Instrument } \\
\text { type }\end{array}$ & Detector type \\
\hline Instrument 1 & 27 & 4 & Survey meter & $\begin{array}{l}\text { Internal ionization } \\
\text { chamber }\end{array}$ \\
\hline Instrument 2 & 44 & 4 & Survey meter & $\begin{array}{l}\text { Internal pressurized } \\
\text { ionization chamber }\end{array}$ \\
\hline Instrument 3 & 33 & 3.5 & $\begin{array}{l}\text { Electronic } \\
\text { personal } \\
\text { dosimeter }\end{array}$ & $\begin{array}{l}\text { Silicon } \\
\text { semiconductor }\end{array}$ \\
\hline
\end{tabular}

\section{NCLS RP-1 Method Analysis of NIST GRSD Data}

\subsection{Data Analysis for Instrument 1}

This instrument displays dose rate in units of $\mathrm{mR} / \mathrm{h}$ and it was calibrated at all ranges from $1 \mathrm{mR} / \mathrm{h}$ to $40000 \mathrm{mR} / \mathrm{h}$ for a total of 10 different dose rate values for each calibration date. These 10 dose rate values are considered as a single measurement for each unit at a given time, i.e. all 10 measurements would have to be in-tolerance. In Fig. 1 it can be observed that one point (shaded gray) for one of the units (at $400 \mathrm{mR} / \mathrm{h})^{1}$ is out of range, i.e. in this case the unit is considered out-of-tolerance. Looking closely at the raw data entries, it is possible that this out-of-range value is just a mistake in the data entry. Based in the NCLS RP-1 method outliers such as this value could be removed from the data analysis, see Ref. [2]. For the purpose of illustrating how the method works this point was kept in the data analysis. As described in the NCLS RP-1 method, the data of all 44 units' serial numbers were combined and provided the following input data to determine the calibration interval:

- The instrument is calibrated only when it is out of tolerance (in Fig. 1 values are shown as in tolerance or out of tolerance)

\footnotetext{
${ }^{1}$ NIST does not endorse the use of non-SI units. This paper uses non-SI units because it addresses the actual instruments' displayed unit.
} 
- $\quad$ Number of Tests $=80$

- $\quad$ Number In-Tolerance $=79$

- Observed Reliability $=99 \%$

- Current Interval $=2.36$ years (to illustrate the use of a longer calibration interval, used the first time one reading was out of tolerance instead of the 1-year calibration interval)

- Longest Interval $=3.71$ years (time of available data)

- Reliability Target $=95 \%$

- Interval Change Confidence $=95 \%$

The results obtained for this input data are:

- Interval Rejection Confidence $=89.74 \%$

- Upper Confidence Limit $=99.97 \%$

- Lower Confidence Limit $=93.23 \%$

- Recommended Interval $=2.36$ years (the recommended interval would have been 1 year if the entered current interval was 1 year)

As the $95 \%$ Interval Change Confidence is contained within the upper and lower confidence limits, the current interval is accepted, then the recommended recalibration interval is the same, 2.36 years (Case 1). If the number of in-tolerance was 80 instead of 79 then the recommended interval is 4.45 years (Case 2). In addition, using the same parameters but changing the current interval to 1 year and the number in-tolerance to 80 (Case 4), then the recommended interval is 2 years (this will be the case where a 1-year calibration interval is used and all readings are in-tolerance, the out-of-tolerance value is considered to be a mistake in the data entry or an outlier). Table 3 shows a summary of the cases described here (Case 1, Case 2 and Case 4) plus some additional ones where the Reliability Target and Interval Change Confidence were reduced to $90 \%$. In Case 5 Reliability Target is reduced to $90 \%$ instead of $95 \%$, the rest of the values are the same as in Case 3. In this case, by reducing the Reliability Target the recommended interval changed from 1 year to 2 years. In Case 6 both the Reliability Target and Interval Change Confidence were reduced to $90 \%$. In this case, the recommended interval changed from 1 year to 2 years. 


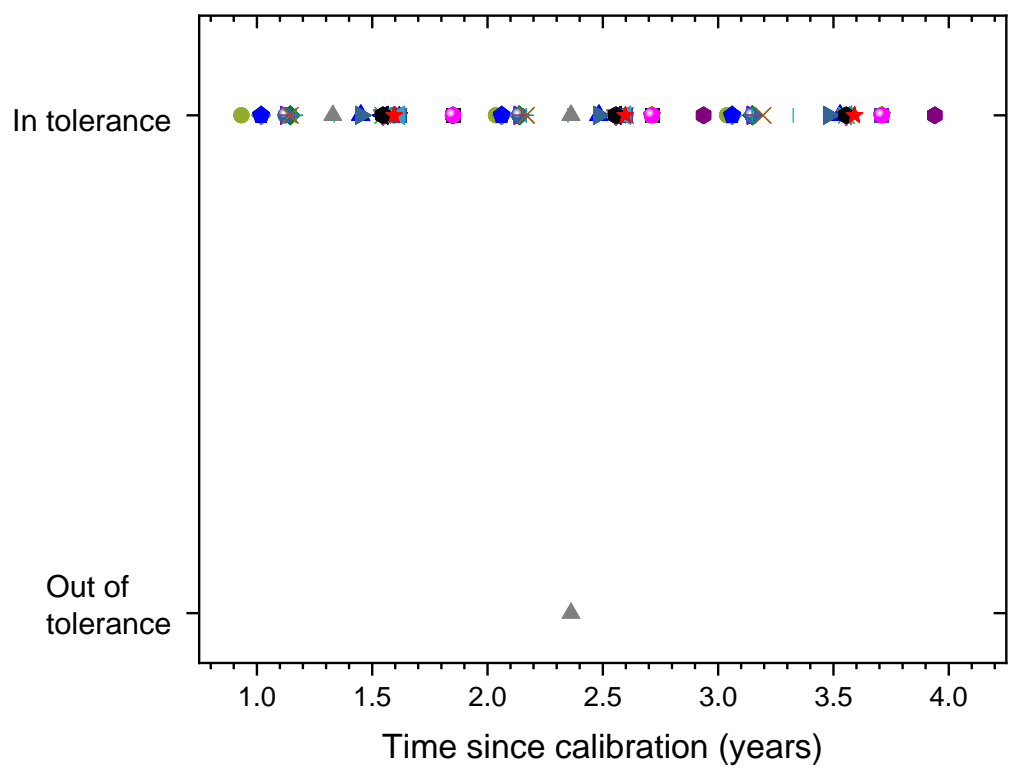

Fig. 1: Instrument 1 in-tolerance readings as a function of time for 3.71 years for all units. The different color symbols represent the different units. There are 10 dose rate values per calibration for each unit. The gray triangle at zero values, is one exposure rate value out-of-tolerance while the other 9 values are in tolerance.

Table 3: Example cases for the Instrument 1

\begin{tabular}{|l|l|l|l|l|l|l|}
\hline & Case 1 & Case 2 & Case 3 & Case 4 & Case 5 & Case 6 \\
\hline $\boldsymbol{R}_{\text {targ }}$ & $95 \%$ & $95 \%$ & $95 \%$ & $95 \%$ & $90 \%$ & $90 \%$ \\
\hline Min allowed interval & 0 & 0 & 0 & 0 & 0 & 0 \\
\hline Max allowed interval & 10000 & 10000 & 10000 & 10000 & 10000 & 10000 \\
\hline $\begin{array}{l}\text { Interval change } \\
\text { confidence }\end{array}$ & $95 \%$ & $95 \%$ & $95 \%$ & $95 \%$ & $95 \%$ & $90 \%$ \\
\hline Current interval & 2.36 years & 2.36 years & 1 year & 1 year & 1 year & 1 year \\
\hline Longest interval & 3.71 years & 3.71 years & 3.71 years & 3.71 years & 3.71 years & 3.71 years \\
\hline Number of tests & 80 & 80 & 80 & 80 & 80 & 80 \\
\hline $\begin{array}{l}\text { Number in-tolerance } \\
\text { confidence }\end{array}$ & 79 & 80 & 79 & 80 & 79 & 79 \\
\hline $\begin{array}{l}\text { Observed reliability } \\
\text { Interval rejection }\end{array}$ & $98.75 \%$ & $100 \%$ & $98.75 \%$ & $100 \%$ & $98.75 \%$ & $98.750 \%$ \\
\hline
\end{tabular}




\begin{tabular}{|l|l|l|l|l|l|l|}
\hline $\begin{array}{l}\text { Upper confidence } \\
\text { limit }\end{array}$ & $99.97 \%$ & $100 \%$ & $99.97 \%$ & $100 \%$ & $99.97 \%$ & $99.94 \%$ \\
\hline $\begin{array}{l}\text { Lower confidence } \\
\text { limit }\end{array}$ & $93.23 \%$ & $95.49 \%$ & $93.23 \%$ & $95.49 \%$ & $93.23 \%$ & $94.21 \%$ \\
\hline $\begin{array}{l}\text { Recommended } \\
\text { interval }\end{array}$ & 2.36 years & 4.45 years & 1 year & 2 years & 2 years & 2 years \\
\hline
\end{tabular}

\subsection{Data Analysis for Instrument 2}

This instrument displays dose rate in units of $\mathrm{mR} / \mathrm{h}$ and it was calibrated at all ranges from $0.04 \mathrm{mR} / \mathrm{h}$ to $4000 \mathrm{mR} / \mathrm{h}$ for a total of 11 different dose rate values for each calibration date. As the data of all units' serial numbers were combined, that provided the following input data to determine the calibration interval:

- The instrument is calibrated only when it is out of tolerance (in Fig. 2 values are shown as in tolerance or out of tolerance)

- $\quad$ Number of Tests $=128$

- $\quad$ Number In-Tolerance $=107$

- Current Interval $=1$ years $(\mathrm{NRC}$ required calibration time interval $)$

- $\quad$ Longest Interval $=3.96$ years (time of available data)

- Observed Reliability $=83.59 \%$

- $\quad$ Reliability Target $=95 \%$

- Interval Change Confidence $=95 \%$

The results obtained for this input data are:

- Interval Rejection Confidence $=100 \%$

- Upper Confidence Limit $=89.55 \%$

- $\quad$ Lower Confidence Limit $=76.02 \%$

- $\quad$ Recommended Interval $=1$ years

As the $95 \%$ Interval Rejection Confidence is not contained within the upper and lower confidence limits the current interval is accepted, then the recommended recalibration interval is 1 year. If using the same values but changing the Reliability Target to $64 \%$ and the Interval Change Confidence to $30 \%$ then the recommended interval would be 2 years.

If GRSD's acceptance range is changed from $\pm 20 \%$ to $\pm 30 \%$, then all the instruments' readings would have been in range except for one at 2.31 years (see Fig. 4). Using the same parameters listed above but changing the number in-tolerance to 127 , then the recommended interval would be 2 years. If in addition, the current interval was changed to 2.31 years then the recommended interval would be 4.62 years. 


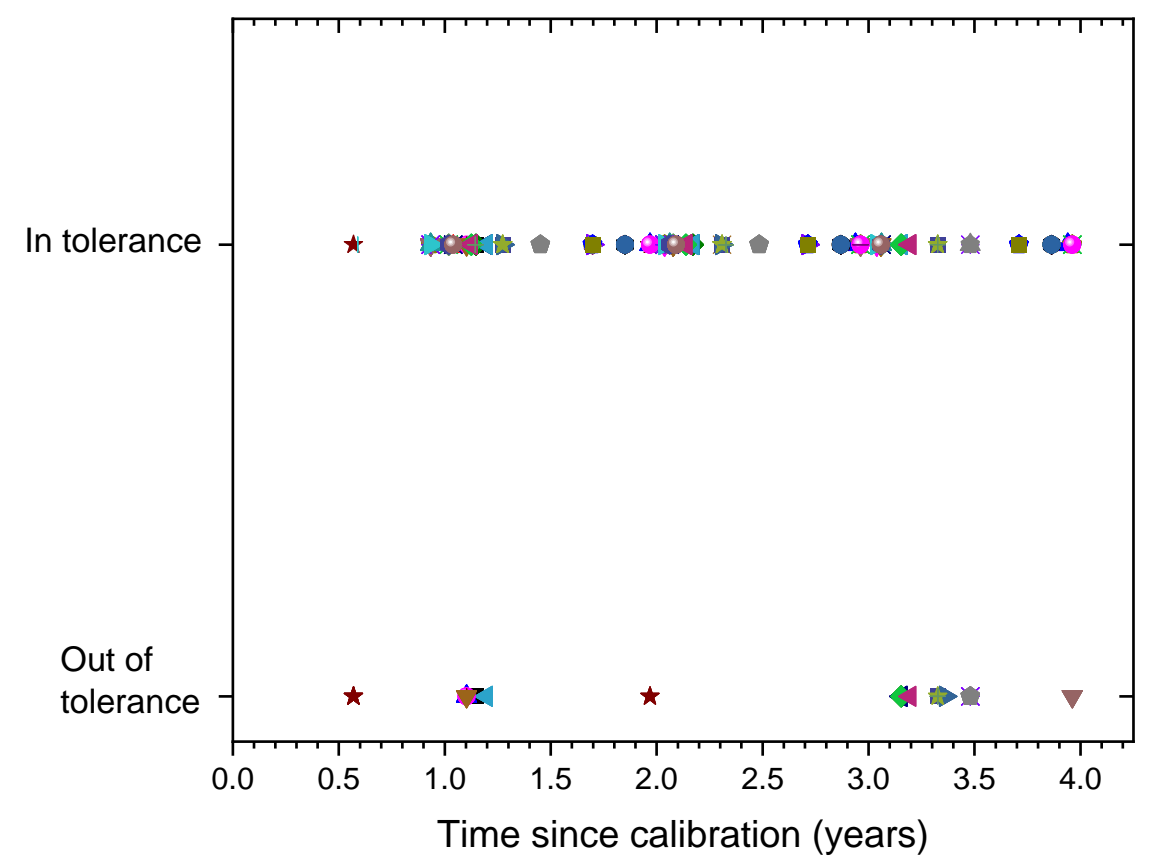

Fig. 2: Instrument 2 in tolerance readings as a function of time for 3.96 years for all units. The different color symbols represent the different units. There are 11 dose rate values per calibration for each unit.

\subsection{Data Analysis for Instrument 3}

This instrument displays dose rate in units of $\mathrm{mrem} / \mathrm{h}$ and it was calibrated at all ranges from $2 \mathrm{mrem} / \mathrm{h}$ to $8000 \mathrm{mrem} / \mathrm{h}$ for a total of 8 different dose rate values for each calibration date. The data of all units' serial numbers were combined, providing the following input data to determine the calibration interval:

- The instrument is calibrated only when it is out of tolerance (in Fig. 3 values are shown as in tolerance or out of tolerance)

- $\quad$ Number of Tests $=47$

- $\quad$ Number In-Tolerance $=47$

- Observed Reliability $=100 \%$

- Current Interval = 1 year

- Longest Interval $=3.14$ years (time of available data)

- Reliability Target $=95 \%$

- Interval Change Confidence $=95 \%$

The results obtained for this input data are:

- Interval Rejection Confidence $=91.03 \%$ 
- Upper Confidence Limit $=100 \%$

- Lower Confidence Limit $=92.45 \%$

- Recommended Interval $=1$ years (under these conditions the recommended interval would have changed to 2 years if there were 72 tests and 72 in-tolerance)

As the $95 \%$ interval change confidence is contained within the upper and lower confidence limits, the current interval is accepted, therefore the recommended recalibration interval is 1 years. If we assume that the Current interval $=3$ years and Longest interval $=3.14$ years, then the Recommended interval would be 3 years.

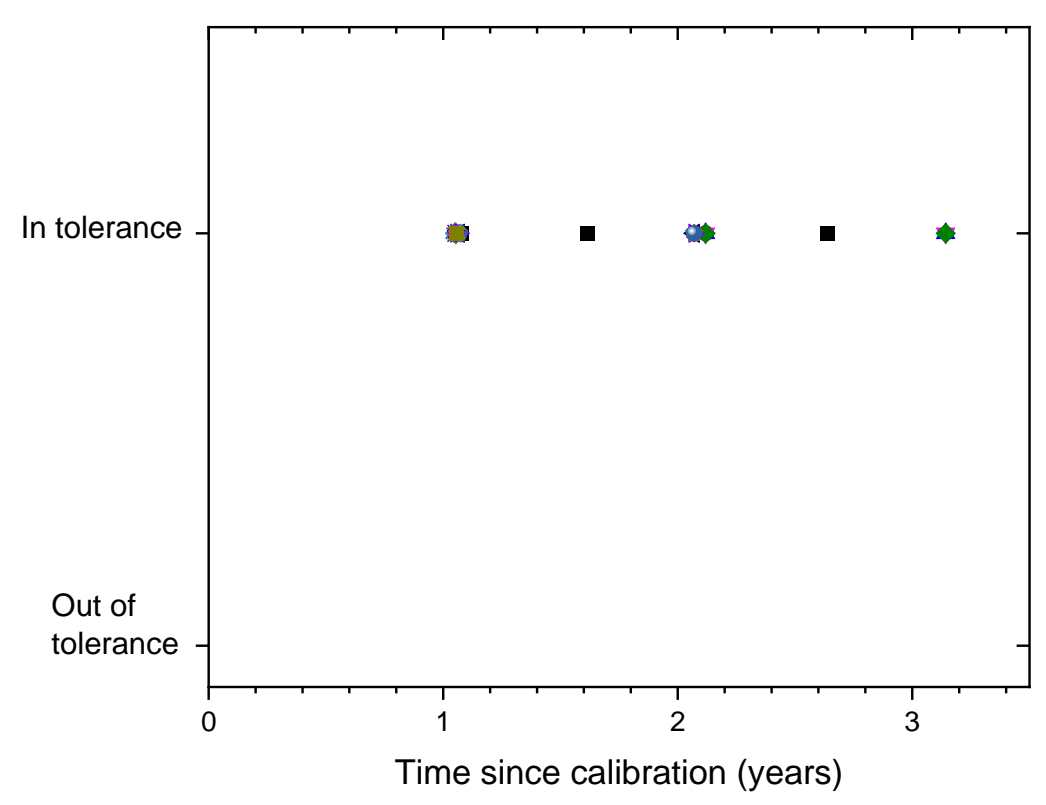

Fig. 3: Instrument 3 in tolerance readings as a function of time for 3.14 years for all units. The different color symbols represent the different units. There are 8 dose rate values per calibration for each unit.

\subsection{Exposure Rate Readings for Instrument 2}

Fig. 4 shows the percent difference between the exposure rate readings from the Instrument 2 survey meter data (same data as in Sec. 4.2 used to apply the NCSL RP-1 method) and the applied exposure rate using the reference beams. This plot combines all the units of the same model. As mentioned before, the Instrument 2 survey meter was exposed to different exposure rate values for each measurement date using NIST reference beams. The lines in Fig. 4 represent the $\pm 30 \%, \pm 20 \%$ and the $0 \%$ difference between the values displayed by the instrument and the applied radiation field. The $\pm 20 \%$ acceptance range is the currently used acceptance rate for this type of instruments [1] that determines when the instrument response is in-tolerance or out-tolerance. From Fig. 4 can be observed that if the selected acceptance range would have been $\pm 30 \%$, instead of $\pm 20 \%$, then the number of out-of-tolerance readings would have been less. As mentioned before, the Instrument 2 instruments were 
adjusted in some cases when the readings were within the acceptance range, in these cases the measurements were recorded as out-of-tolerance. This explains the smaller number of points outside the $\pm 20 \%$ acceptance range in Fig. 4 compared to the out-of-tolerance points in Fig. 2. Looking closely at the raw data entries for the Instrument 2 instrument, it is possible that the out-of-range value at approximately $-90 \%$ is just a mistake in the data entry. The change in the acceptance range would have change the recommended calibration interval when using the NCSL RP-1 method). For some applications the acceptance range may be set by regulations in other cases regulations may not exist so the value may be adjusted depending on the application and needs.

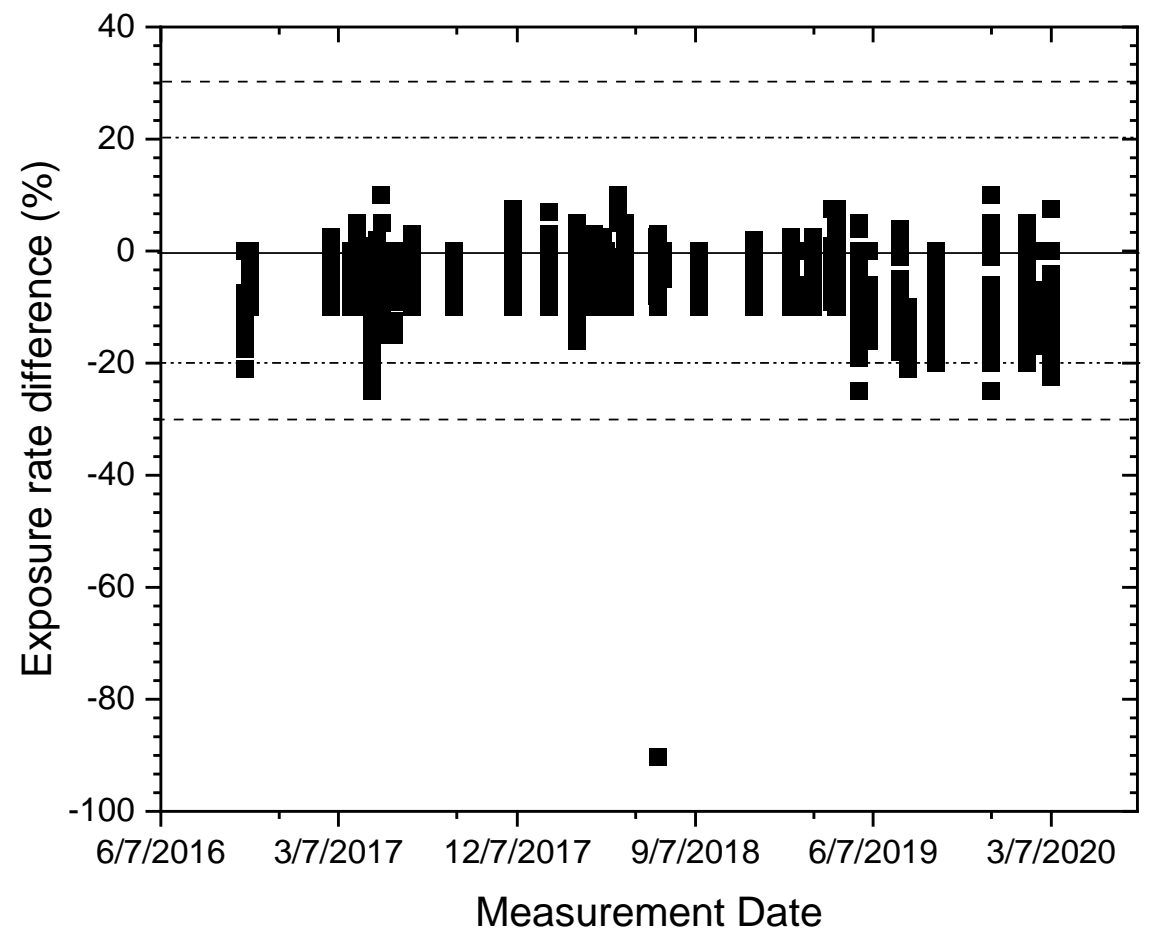

Fig. 4: Exposure rate difference between the instrument measured value and the radiation field used for the measurements expressed in percent. The lines represent the $0 \%$, the $\pm 20 \%$ and the $\pm 30 \%$ differences. The points outside the $\pm 20 \%$ lines and at $-90 \%$ represent some of the out-of-tolerance values.

\section{ANSI N323AB Control Charts}

In addition to the method described in the NCSL RP-1 document, the ANSI N323AB standard provides additional methods to determine the performance of radiation detection instruments. Among these methods are control charts. This method applies to individual instruments rather than combining units of a given instrument model. This method allows the user to follow the instrument performance over time potentially allowing to extend the calibration time, so the instruments can be calibrated or sent out for calibration when the instrument response it out of range. The tracking of the instrument response over time needs to start as soon as the instrument is calibrated or purchased (assumes that the instrument is calibrated when 
purchased) to ensure the instrument response is within range at the starting time. This method allows for the use of uncalibrated check sources instead of calibrated radiation fields that make use of large radioactive sources, but it can also be performed using calibrated radiation fields (or reference beams) if one is readily available.

How to develop control charts and plots?

- At the time an instrument is purchased or calibrated place the detector in front of a radioactive source at a fixed source to detector distance.

- The relative position between the detector and source needs to be reproducible. It is recommended that a positioning jig is built or purchased such that the detector and the source are fixed relative to each other.

- A chart and data sheet should be created to record the instrument response to the source and develop the acceptable tolerance range for that source and instrument.

- The instrument may display different units, so the control chart measurements should always be performed using the same settings. The source activity at the time of the measurements shall be decay corrected to allow the comparison in the instrument readings.

- The instrument is recalibrated if the instrument reading differs from the initial reading by a given predetermined value designated by the individual agency depending on their requirements.

- If the instrument is dropped or shocked while being used, verify that the readings are within the same range. Both the count rate and the integrated rate may need to be verified.

- If the instrument has spectrometric capabilities then a radionuclide (or a mix of radionuclides) emitting low and high gamma-rays needs to be used to verify the full energy peak efficiency, the full width half maximum (FWHM) and the energy calibration.

\subsection{Examples of control charts for instrument providing exposure or dose rate or count rate readings}

Based on the data provided in Sec. 4.4 it would be possible to plot for each instrument the exposure or dose or count rate readings as a function of measurement time. Spread sheets or any other similar application may be used to record the instrument response data over time. When recording this type of data, it is recommended at a minimum to record the following data elements:

- Date and time of measurements

- Source used

- Radiation field used for the measurements (expressed in count rate, $\mathrm{mR} / \mathrm{h}$ or $\mathrm{mrem} / \mathrm{h}$ depending of the units displayed by the instrument)

- Radiation field measured by the instrument (expressed in $\mathrm{mR} / \mathrm{h}$ or $\mathrm{mrem} / \mathrm{h}$ depending of the units displayed by the instrument)

- Record if the instrument reading was in-tolerance (yes/no) 
- Record if the instrument was adjusted or left as is (this is only relevant during a calibration procedure. For example, if a user identifies that an instrument's response is outside of the $20 \%$ tolerance, the action should be re-calibration to a calibrated reference standard rather than an adjustment associated with a check source)

- Operator name

- Instrument model and serial number

\subsection{Examples of control charts and plots for instrument with radionuclide identification capabilities}

The examples shown below are control plots obtained for a high purity germanium (HPGe) detector for which the initial calibration was performed in 2009 and the detector performance was monitored monthly for more than 10 years. For this detector the full energy peak efficiency, the FWHM and the photopeak energy were monitored over time. An application or program is required to extract the necessary information from an HPGe spectrum to determine these parameters (e.g., Genie 2000, GammaVision, etc. ${ }^{2}$. The plots below show the detector monthly response during the year 2019 (see Fig. 5 through Fig. 8). The values measured in 2009 are displayed as time zero. Two different ${ }^{57} \mathrm{Co}$ and ${ }^{60} \mathrm{Co}$ sources were used to perform the efficiency measurements in 2009 and 2019, the activity value for the 2009 source has a larger uncertainty that the 2019 source activity value. As the measurement geometry is reproducible and the source activities are well known the instrument response can be followed over a long period of time even when the source was replaced or time as the sources decay. For this application the allowed variability in the full energy peak efficiency was $\pm 1.5 \%$, for other applications this value can be larger or smaller, it will depend on the accuracy requirements for the use-case of the measurements. Changes in the values of the full energy peak efficiency can be attributed to changes in the radioactive source position relative to the detector or changes in the detector crystal or electronics. Changes in the FWHM are also attributed to changes in the detector crystal or electronics. Drifts in the photopeak energy can be due to temperature changes and changes in the detector electronics, this can easily be periodically adjusted following the manufacturer specification or the procedures listed in the ANSI N42.14 [5] or the IEC 61452 [6] standards. It is recommended to check the detector energy calibration prior to performing any measurement, this can be performed by acquiring a background spectrum and locating the main gamma-ray lines that are present in the background or by using a check source. Spread sheets were used to summarize the data as a function of time.

\footnotetext{
${ }^{2}$ Certain commercial equipment, instruments, or materials are identified in this paper to foster understanding. Such identification does not imply recommendation by the National Institute of Standards and Technology, nor does it imply that the materials or equipment identified are necessarily the best available for the purpose.
} 


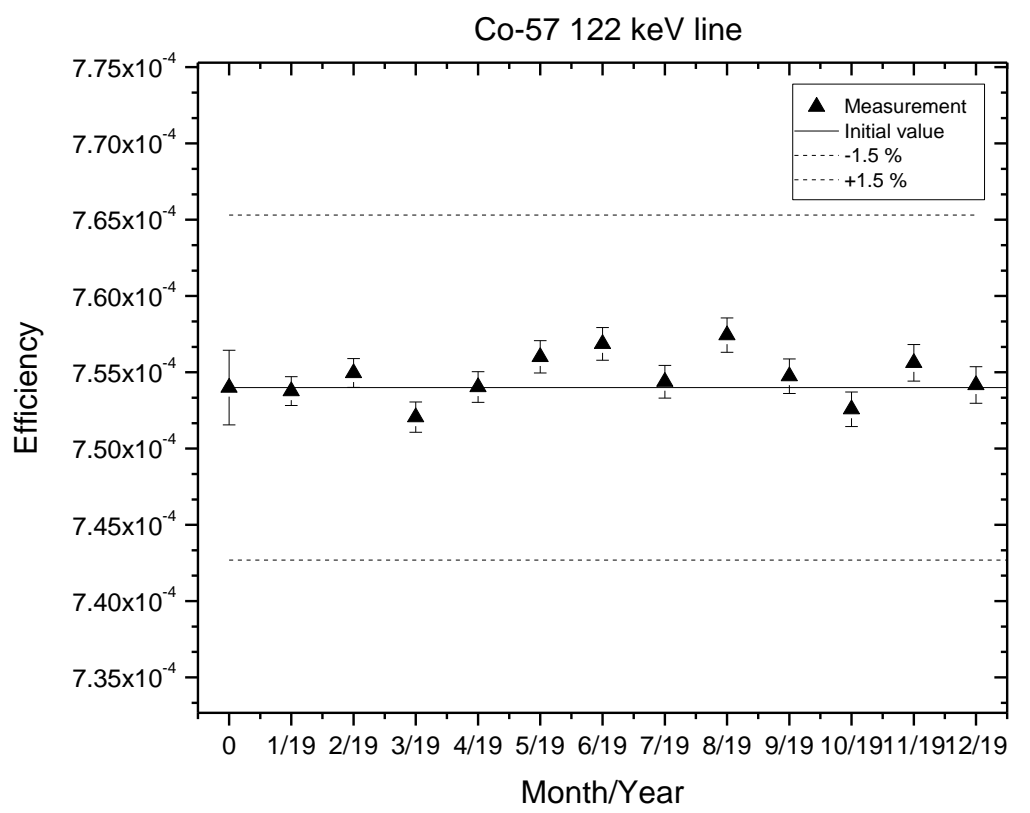

Fig. 5: Full energy peak efficiency for the $122 \mathrm{keV}$ gamma-ray from the ${ }^{57} \mathrm{Co}$ emission. The triangles represent the measured values over time for the year 2019. The time zero and the solid line represent the initial measurement performed in 2009 . The dotted lines represent the $\pm 1.5 \%$ values of the initial value.

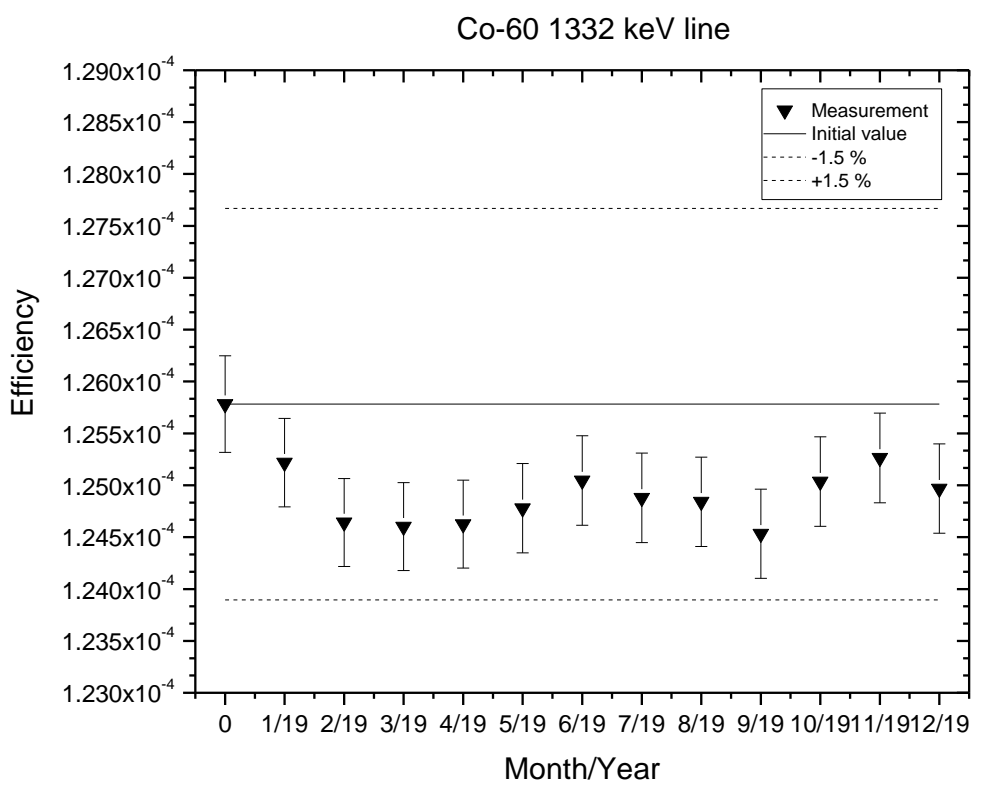

Fig. 6: Full energy peak efficiency for the $1332 \mathrm{keV}$ gamma-ray from the ${ }^{60} \mathrm{Co}$ emission. The triangles represent the measured values over time for the year 2019. The time zero and the solid line represent the initial measurement performed in 2009. The dotted lines represent the $\pm 1.5 \%$ values of the initial value. 


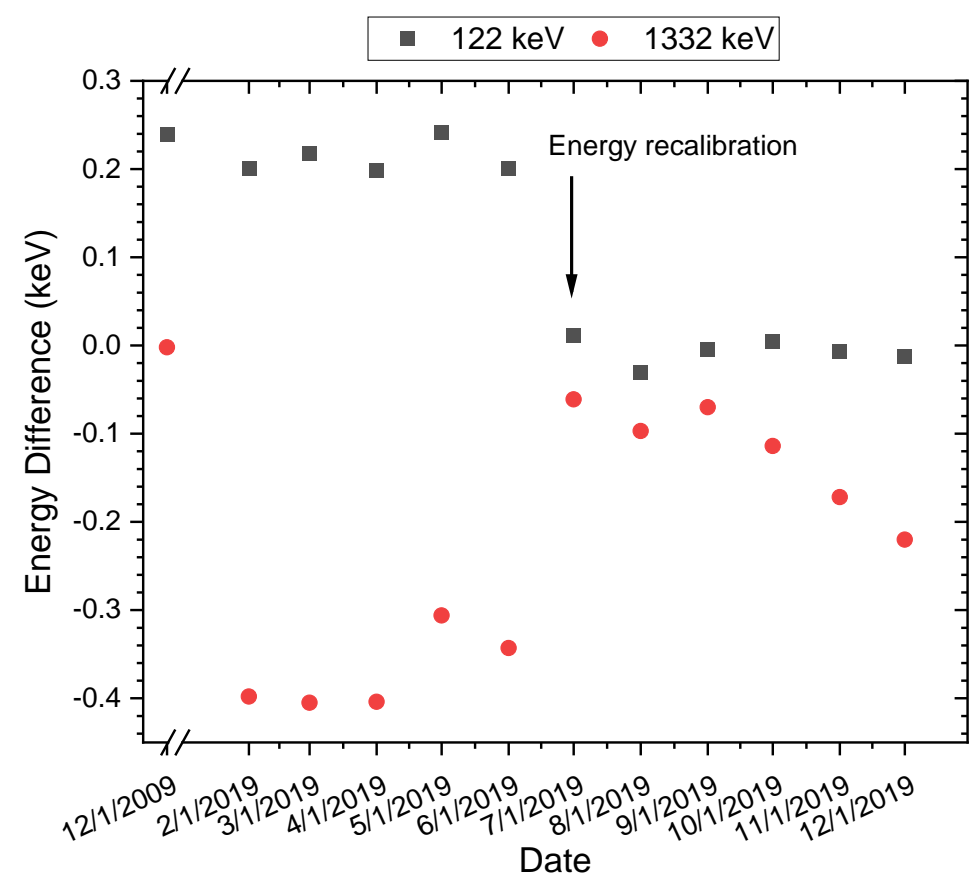

Fig. 7: Energy difference between the measured and evaluated values for the main gamma-ray lines for ${ }^{57} \mathrm{Co}$ and ${ }^{60} \mathrm{Co}$. The solid line represents zero difference.

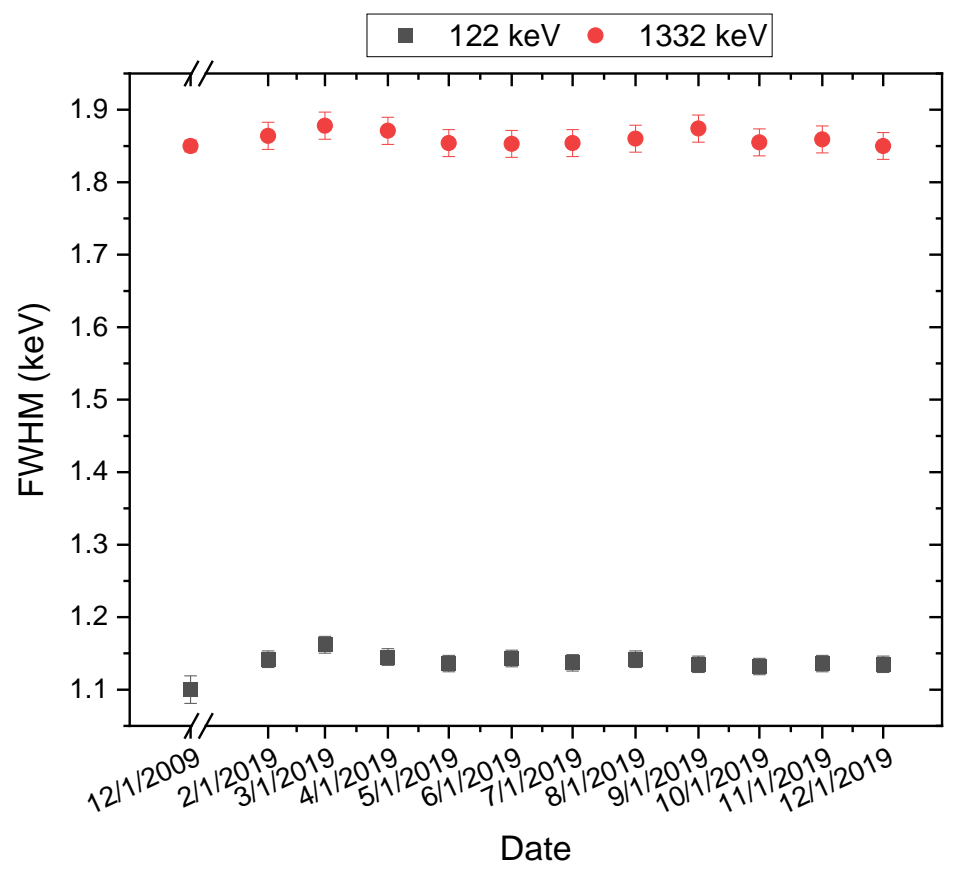

Fig. 8: Full width half maximum (FWHM) for the main gamma-ray lines for ${ }^{57} \mathrm{Co}$ and ${ }^{60} \mathrm{Co}$. 


\section{Discussions}

\subsection{For instruments providing dose, exposure or count rate readings}

As shown in the previous sections for this type of instruments different methods can be used to track the instrument performance over time. These methods have different pros and cons. The method described in the NCSL RP-1 document allows for the combination of different units of the same instrument model, but it requires a significant number of measurements (this number depends on the input user criteria, see Sec. 2) in order to reliably apply it. This might be a useful method when users have a large number of identical instruments in their inventory. The results from this method depend on several parameters as described in Sec. 4, in addition, it is important to note that the recommended interval is dependent on the current calibration interval.

The use of control charts is also possible but as it applies to individual instruments it might not be advantageous for large instrument inventories. Both methods (i.e., NCSL RP-1 and control charts) can be applied using check source or calibrated radiation fields. The disadvantage of using check sources is that it is not possible to verify the instrument response at high dose rate values. The disadvantage of using calibrated radiation beams is that they may not be easily accessible to all instrument users.

\subsection{For instrument with radionuclide identification capabilities}

Spectroscopic type of instruments cannot measure very strong sources as when the dead time is too high the instrument stops responding to the radiation source and the crystal can be damaged. In this case, a small activity check source can be used to follow the instrument performance using control charts. In addition, the cost of this type of instruments is higher than gross count instruments so users may have a smaller number of instruments in their inventory.

\subsection{Data quality objectives}

No matter the type of instrument, the user needs to define the data quality objectives. For control charts users need to define the acceptance range over which the instrument can be reliably used. When the NCSL RP-1 method is used the Reliability Target, Out of Tolerance Rate Target, Display Precision, Minimum Allowed Interval, Maximum Allowed Interval and Interval Change Confidence need to be defined. Different results will be obtained if different input values are used. The observed reliability (\% in-tolerance) is defined by the acceptance instrument reading range. This acceptance range may vary depending on the accuracy required for the user's application and the applicable regulations (e.g., $\pm 10 \%$ ANSI 323AB standard for high dose rate measurements, $\pm 20 \%$ used by NRC regulation, $\pm 30 \%$ used in homeland security standards for interdiction applications and for accreditation of personal dosimeters).

\subsection{Logbook vs electronic records}

NIST retains paper logbook records for all their instruments dating from decades ago. These records were not used in this data analysis as it was too labor intensive (time consuming and different data format) to transfer it to a useable electronic form. The use of the NCSL RP-1 method or control charts require electronic data, so for users who would want to apply the analysis it is recommended to record calibration data directly in an electronic form or database. The additional advantage of recording the data in an electronic form is that the format can be maintained over time and even additions or changes to the spread sheet or database can be easily addressed. 


\subsection{Analysis of NIST GRSD data}

The data in Fig. 2 and Fig. 4 belong to the same instruments. In Fig. 4 the acceptance range was set to $\pm 20 \%$ based on NRC regulations [1]. One point was out of range by close to $100 \%$ at a dose rate of $400 \mathrm{mrem} / \mathrm{h}$, this seems to be a mistake in the data entry. If the acceptance range was $\pm 30 \%$ instead of $\pm 20 \%$ then only 1 point would have been out-of-tolerance. This change would have resulted in a recommended calibration interval of 2 years (for the same input parameters listed in Sec. 4.2).

\section{Conclusions}

From the NIST GRSD data it can be observed that when using the method described in the NCSL RP-1 document the recalibration interval can be increased when the number of intolerance readings is close to the number of measurements performed (observed reliability close to $100 \%$, when high reliability target and interval change confidence values are used). This method allows for the combination of several units of the same instrument model. The ANSI N323AB standards describes several methods to determine the calibration interval among which are the NCSL RP-1 method and the use of control charts. Control charts may be used to track the individual performance of a given instrument. Acceptance ranges may be chosen depending on the user needs or regulatory requirements as applicable.

Tracking the response of instruments over time allows the user to determine the instrument reliability as well as to determine when the instrument response is outside the acceptance range and requires recalibration. Not all devices behave the same way, so it is not possible to have a universally appropriate recalibration time unless such time is set by a regulatory agency that requires a predefined recalibration time. Currently, regulatory agency values apply to certain types of instruments and for specific applications. Some applications may not be under any regulations and users may choose the tolerance range based on the instrument use and required accuracy of the measurements.

When trying to determine the optimal recalibration interval it is necessary to perform measurements over several years or to perform more frequent measurements (e.g., less than 1 year) in order to have high confidence that a longer interval will achieve the target reliability. The number of points required to make a calibration interval determination will depend mainly on the number in-tolerance measurements, the reliability target and the interval change confidence required by the user.

\section{References}

[1] NRC Regulations - 10 CFR Part 34. Licenses for Industrial Radiography and Radiation Safety Requirements for Industrial Radiography Operations. https://www.nrc.gov/reading-rm/doc-collections/cfr/part034/

[2] National Conference of Standards Laboratories (NCSL) International (2010), Recommend Practice RP-1: Establishment and Adjustment of Calibration Intervals.

[3] ANSI N323AB. American National Standard for Radiation Protection Instrumentation Test and Calibration, Portable Survey Instruments.

[4] Tool for A3 method described in NCSL RP-1 http://www.isgmax.com/calint_freeware.htm 
[5] ANSI N42.14. American National Standard for Calibration and Use of Germanium Spectrometers for the Measurement of Gamma-Ray Emission Rates of Radionuclides.

[6] IEC 61452. Nuclear Instrumentation - Measurement of Gamm-Ray Emission Rates of Nuclide - Calibration and Use of Germanium Spectrometers. 